\title{
4
}

\section{Adaptive Feedforward Control for Gust Loads Alleviation}

\author{
Jie Zeng ${ }^{1}$, Raymond De Callafon ${ }^{2}$ and Martin J. Brenner ${ }^{3}$ \\ ${ }^{1}$ ZONA Technology, Inc. \\ ${ }^{2}$ University of California, San Diego \\ ${ }^{3}$ NASA Dryden Flight Research Center \\ USA
}

\section{Introduction}

Active control techniques for the gust loads alleviation/flutter suppression have been investigated extensively in the last decades to control the aeroelastic response, and improve the handling qualities of the aircraft. Nonadaptive feedback control algorithms such as classical single input single output techniques (Schmidt \& Chen, 1986), linear quadratic regulator (LQR) theory (Mahesh et al., 1981; Newsom, 1979), eigenspace techniques (Garrard \& Liebst, 1985; Leibst et al., 1988), optimal control algorithm (Woods-Vedeler et al., 1995), $H_{\infty}$ robust control synthesis technique (Barker et al., 1999) are efficient methods for the gust loads alleviation/flutter suppression. However, because of the time varying characteristics of the aircraft dynamics due to the varying configurations and operational parameters, such as fuel consumption, air density, velocity, air turbulence, it is difficult to synthesize a unique control law to work effectively throughout the whole flight envelope. Therefore, a gain scheduling technique is necessary to account for the time varying aircraft dynamics.

An alternative methodology is the feedforward and/or feedback adaptive control algorithms by which the control law can be updated at every time step (Andrighettoni \& Mantegazza, 1998; Eversman \& Roy, 1996; Wildschek et al., 2006). With the novel development of the airborne LIght Detection and Ranging (LIDAR) turbulence sensor available for an accurate vertical gust velocity measurement at a considerable distance ahead of the aircraft (Schmitt, Pistner, Zeller, Diehl \& Navé, 2007), it becomes feasible to design an adaptive feedforward control to alleviate the structural loads induced by any turbulence and extend the life of the structure. The adaptive feedforward control algorithm developed in (Wildschek et al., 2006) showed promising results for vibration suppression of the first wing bending mode. However, an unavoidable constraint for the application of this methodology is the usage of a high order Finite Impulse Response (FIR) filter. As a result, an overwhelming computation effort was needed to suppress the structural vibration of the aircraft.

In this chapter, an adaptive feedforward control algorithm where the feedforward filter is parameterized using orthonormal basis expansions along with a recursive least square algorithm with a variable forgetting factor is proposed for the feedforward compensation of gust loads. With the use of the orthonormal basis expansion, the prior flexible modes information of the aircraft dynamics can be incorporated to build the structure of the feedforward controller. With this strategy, the order of the feedforward filter to be estimated 
can be largely reduced. As a result, the computation effort is greatly decreased, and the performance of the feedforward controller for gust loads alleviation will be enhanced. Furthermore, an FFT based PolyMAX identification method and the stabilization diagram program (Baldelli et al., 2009) are proposed to estimate the flexible modes of the aircraft dynamics.

The need for an integrated model of flight dynamics and aeroelasticity is brought about by the emerging design requirements for slender, more flexible and/or sizable aircraft such as the Oblique Flying Wing (OFW), HALE, Sensorcraft and morphing vehicles, etc. Furthermore, a desirable unified nonlinear simulator should be formulated in principle by using commonly agreeable terms from both the flight dynamics and aeroelasticity fields in a consistent manner.

A unified integration framework that blends flight dynamics and aeroelastic modeling approaches with wind-tunnel or flight-test data derived aerodynamic models has been developed in (Baldelli \& Zeng, 2007). This framework considers innovative model updating techniques to upgrade the aerodynamic model with data coming from CFD/wind-tunnel tests for a rigid configuration or data estimated from actual flight tests when flexible configurations are considered.

Closely following the unified integration framework developed in (Baldelli \& Zeng, 2007), an F/A-18 Active Aeroelastic Wing (AAW) aeroelastic model with gust perturbation is developed in this chapter, and this F/A-18 AAW aeroelastic model can be implemented as a test-bed for flight control system evaluation and/or feedback/feedforward controller design for gust loads alleviation/flutter suppression of the flexible aircraft.

The outline of the chapter is as follows. In Section II, a feedforward compensation framework is introduced. Section III presents the formulation of the orthonormal finite impulse filter structure. A brief description of a frequency domain PolyMAX identification method is presented in Section IV. In Section V, a recursive least square estimation method with variable forgetting factor is discussed. Section VI includes the development of a linear F/A-18AAW aeroelastic model and the application of the adaptive feedforward controller to F/A-18 AAW aeroelastic model.

\section{Basic idea of the feedforward controller}

In order to analyze the design of the feedforward controller, $F$, consider the simplified block diagram of structural vibration control of a single input signal output (SISO) dynamic system depicted in Fig. 1. The gust perturbation, $w(t)$, passes through the primary path, $H$, the body of the aircraft, to cause the structural vibrations. Mathematically, $H$ can be characterized as the model/transfer function from the gust perturbation to the accelerometer sensor position. The gust perturbation, $w(t)$, can be measured by the coherent LIDAR beam airborne wind sensor. The measured signal, $n(t)$, is fed into the feedforward controller, $F$, to calculate the control surface demand, $u(t)$, for vibration compensations. The structural vibrations are measured by the accelerometers providing the error signal, $e(t) . G$ is the model/transfer function from the corresponding control surface to the accelerometer sensor position, and which is so called the secondary path.

In order to apply the feedforward control algorithm for gust loads alleviation, developing a proper sensor to accurately measure the gust perturbation is crucial for the success of the feedforward control application. As mentioned in (Schmitt, Pistner, Zeller, Diehl \& Navé, 2007), such a sensor should meet the following criteria: 


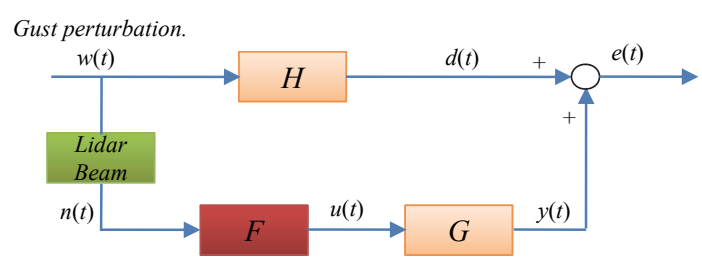

Fig. 1. Block Diagram of the Structural Vibration Control with Feedforward Compensation.

- A feedforward-looking measuring of 50 to $150 \mathrm{~m}$ to ensure that the measured air flow is the one actually affecting the aerodynamics around the aircraft.

- The sensor must be able to measure the vertical wind speed.

- The standard deviation of the wind speed measurement should be small, at least in the range of $[2-4] \mathrm{m} / \mathrm{s}$.

- The sensor must be able to produce reliable signals in the absence of aerosols.

- A good longitudinal resolution (the thickness of the air slice measured ahead).

- A good temporal resolution.

A sensor system that meets these requirements is a so-called short pulse UV Doppler LIDAR, and was developed in (Schmitt, Pistner, Zeller, Diehl \& Navé, 2007). This short pulse UV Doppler LIDAR was successfully applied to an Airbus 340 to measure the vertical gust speed (Schmitt, Rehm, Pistner, Diehl, Jenaro-Rabadan, Mirand \& Reymond, 2007). The authors in (Schmitt, Rehm, Pistner, Diehl, Jenaro-Rabadan, Mirand \& Reymond, 2007) claimed that the system is ready to be used to design feedforward control for gust loads alleviation.

Assuming a perfect gust perturbation signal can be measured via the LIDAR beam sensor, that means, $n(t)=w(t)$, the error signal, $e(t)$, can be described by

$$
e(t)=[H(q)+G(q) F(q)] w(t)
$$

In case the transfer functions in Eq. (1) are known, an ideal feedforward controller, $F(q)=$ $F_{i}(q)$, can be obtained by

$$
F_{i}(q)=-\frac{H(q)}{G(q)}
$$

in case, $F_{i}(q)$, is a stable and causal transfer function. The solution of $F_{i}(q)$ in Eq. (2) assumes full knowledge of $G(q)$ and $H(q)$. Moreover, the filter, $F_{i}(q)$, may not be a causal or stable filter due to the dynamics of $G(q)$ and $H(q)$ that dictate the solution of the feedforward controller, $F_{i}(q)$. An approximation of the feedforward filter, $F_{i}(q)$, can be made by an output-error based optimization that aims at finding the best causal and stable approximation, $F(q)$, of the ideal feedforward controller in $F_{i}(q)$ in Eq. (2).

A direct adaptation of the feedforward controller $F(q, \theta)$ can be performed by considering the parameterized error signal, $e(t, \theta)$,

$$
e(t, \theta)=H(q) w(t)+F(q, \theta) G(q) w(t) .
$$

Defining the signals

$$
d(t):=H(q) w(t), u_{f}(t):=-G(q) w(t)
$$


where $d(t)$ can actually be measured, and $u_{f}(t)$ is called filtered input signal, Eq. (3) is reduced to

$$
e(t, \theta)=d(t)-F(q, \theta) u_{f}(t)
$$

for which the minimization

$$
\min _{\theta} \frac{1}{N} \sum_{t=1}^{N} e^{2}(t, \theta)
$$

to compute the optimal feedforward filter, $F(q, \theta)$, is a standard output-error (OE) minimization problem in a prediction error framework (Ljung, 1999).

The minimization of Eq. (6) for $\lim _{N \rightarrow \infty}$ can be rewritten into the frequency domain expression

$$
\min _{\theta} \int_{\pi}^{-\pi}\left|H\left(e^{j w}\right)+G\left(e^{j w}\right) F\left(e^{j w}, \theta\right)\right|^{2}
$$

using Parceval's theorem (Ljung, 1999). It can be observed that the standard output-error (OE) minimization problem in Eq. (6) can be used to compute the optimal feedforward filter $F(q, \theta)$, provided $d(t)$ and $u_{f}(t)$ in Eq. (4) are available.

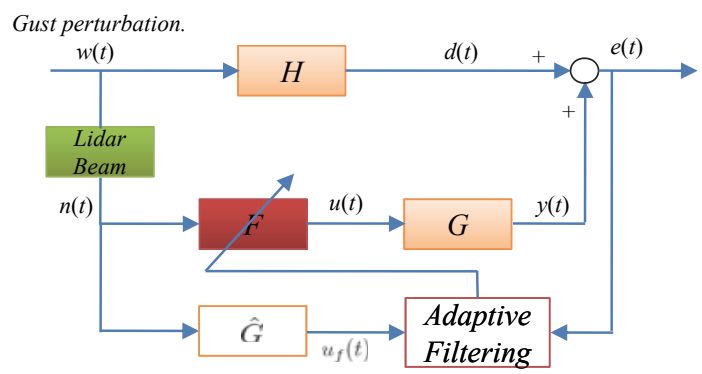

Fig. 2. Block Diagram of the Structural Vibration Control with Adaptive Feedforward Compensation.

For a proper derivation of the adaptation of the feedforward filter, $F$, an approximate model, $\hat{G}$, of the control path, $G$, is required to create the filtered signal, $u_{f}(t)$, for adaptive filtering purpose. The adaptation of the feedforward filter is illustrated in Fig. 2. The filtered signal, $u_{f}(t)$, and the error signal, $e(t)$, are used for the computation of the coefficients of the feedforward filter by the adaptive filtering. Thus, the coefficients of the feedforward filter, $F$, can be updated at each time constant for structural vibration reduction.

\section{ORThonormal Finite Impulse Response (ORTFIR) filter structure}

In general, the feedforward filter, $F$, in Fig. 1 can be realized by adopting both the finite impulse response (FIR) structure as well as the infinite impulse response (IIR) structure. Because the FIR filter incorporates only zeros, it is always stable and it will provide a linear phase response. It is the most popular adaptive filter widely used in adaptive filtering. 
Generally, the discrete time linear time invariant (LTI) FIR filter, $F(q)$, can be presented as:

$$
F(q)=\sum_{k=0}^{L-1} \beta_{k} q^{-k}
$$

where $q^{-1}$ denotes the usual time shift operator, $q^{-1} x(t)=x(t-1)$. Adaptive filter estimation using FIR filters converges to optimal and unbiased estimates irrespective of the coloring of the noise on the output data. However, a FIR filter is usually too simple to model complex system dynamics such as AE/ASE systems with many resonance modes being excited by atmospheric perturbations. As a result, many tapped delay coefficients of the FIR filter are required to approximate the optimal filter. Even though an IIR filter is appealing as an alternative, the inherent stability and bias estimation problems limit the use of an IIR filter for adaptive filtering in aeroservoelastic systems.

To improve the approximation properties of the adaptive filter, $F$, in Fig. 1, the linear combination of tapped delay functions, $q^{-1}$, in the FIR filter of Eq. (8) can be generalized to the following form:

$$
F(q, \theta)=\sum_{k=0}^{L-1} \beta_{k} B_{k}(q)
$$

where $B_{k}(q)$ are generalized (orthonormal) basis functions (Heuberger et al., 1995) that contain some a-priori knowledge on the desired filter dynamics. In other words, the orthonormal basis functions that are used in the parametrization of the ORTFIR filter will be tuned on the fly by taking full advantage of the modal information embedded in the flight data.

Construction of the Orthonormal Basis Sets

The application of orthonormal basis functions to parameterize and estimate dynamical systems has obtained extensive attention in recent years. Different constructions of the orthonormal basis structure has been reported in (Heuberger et al., 1995; Ninness \& Gustafsson, 1997; Zeng \& de Callafon, 2006). It is assumed that the pole locations are already known with the use of the standard open-loop prediction error system identification methods. Suppose the poles $\left\{\xi_{i}\right\}_{i=1,2, \cdots, N}$ are known, an all pass function, $P(q)$, can be created by these poles, and is given as

$$
P(q)=\prod_{i=1}^{N}\left[\frac{1-\xi_{i}^{*} q}{q-\xi_{i}}\right]
$$

Let $(A, B, C, D)$ be a minimal balanced realization of an all pass function, $P(q)$, define the input to state transfer function, $B_{0}(q)=(q I-A)^{-1} B$, then a set of functions, $B_{i}(q)$, can be obtained via

$$
B_{i}(q)=B_{0}(q) P^{i}(q)
$$

and $B_{i}(q)$ has orthogonal property

$$
\frac{1}{2 \pi j} \oint B_{i}(q) B_{k}^{T}(1 / q) \frac{\mathrm{d} q}{q}= \begin{cases}I & i=k \\ 0 & i \neq k\end{cases}
$$

The construction of the orthonormal basis function is illustrated in Fig. 3. It should be noted that if $B_{0}(q)$, includes all information of a dynamical system, then only one parameter, $\beta_{0}$, needs to be estimated to approximate this dynamic system. It means that the parameters 
estimated will directly depend on the a-priori system information injected into the basis functions, $B_{i}(q)$. An important property and advantage of the ORTFIR filter is the knowledge
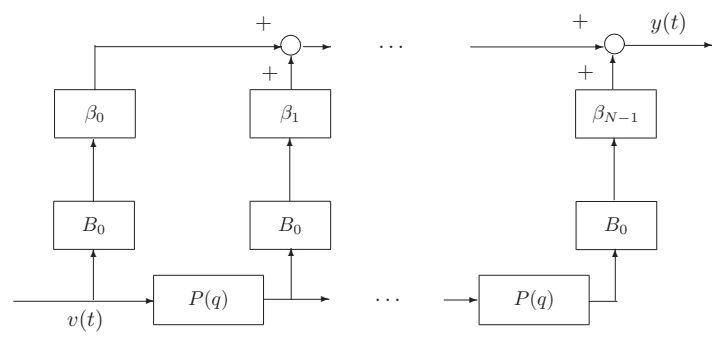

Fig. 3. ORTFIR Filter Topology.

of the (desired) dynamical behavior can be incorporated throughout the basis functions, $B_{i}(q)$. As a result, an accurate description of the filter to be estimated can be achieved by a relatively small number of coefficients.

Case Example: Illustration of the Advantage of Using ORTFIR Filter Over FIR Filter

A 4 degrees-of-freedom (DOF) lumped parameter system is considered to demonstrate the advantage of using ORTFIR filter over FIR filter. An illustration of this 4-DOF lumped parameter system is shown in Fig. 4 , where $k_{i}$ and $c_{i}(i=1, \cdots, 5)$ indicate the system stiffness and damping, respectively and $m_{i}(i=1, \cdots, 4)$ are the masses. The nominal values of these parameters are given as,

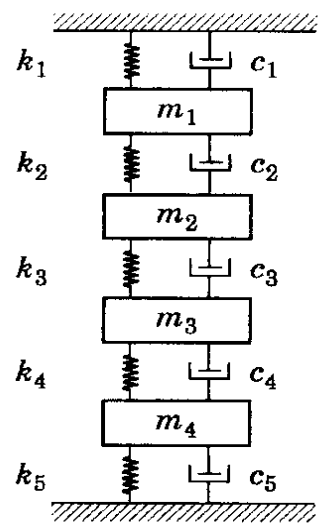

Fig. 4. Lumped Parameter System. 


$$
\begin{aligned}
& m_{1}=m_{2}=m_{3}=m_{4}=1 \\
& k_{1}=k_{3}=k_{5}=1750 \\
& k_{2}=k_{4}=2000 \\
& c_{1}=c_{3}=c_{5}=0.7 \\
& c_{2}=c_{4}=0.8
\end{aligned}
$$

A mathematical model of this lumped system can be easily derived with the use of Newton's second law. The natural frequencies and damping ratios of this lumped system are also obtained. For simplicity purposes, all the units of this 4-DOF lumped system are omitted. This mathematical model is applied in this case example as the real model, and an FIR model and an ORTFIR model will be implemented to approximate this real model, respectively. To facilitate the model estimation using input and output data of the 4-DOF lumped parameter system, a band limited white noise (zero mean) is injected to the 4-DOF lumped parameter system, and an additional band limited white noise (zero mean) is added to the output response to simulate the measurement noise. With the collected input/output data, an FIR filter with varying order is applied to fit the real model, the variance of the simulation error (the difference of the measured and the simulated output) is used to indicate the performance of the FIR filter. Furthermore, the PolyMAX identification method described in Section 4 is applied in this case example to estimate the four physical modes. These estimated physical modes (shown in Table 2, Section 4) are used for the basis function generation of the ORTFIR filter. Finally, an eight order ORTFIR filter is applied to approximate the physical system. The estimation results are shown in Table 1. From Table 1, it is clearly seen that with FIR filter, the optimal FIR filter will be 400th order, with the smallest simulation error 36.18. However, with the simplest eight order ORTFIR filter, the variance of the simulation error is only 13.18, which is almost three times smaller than that of 400th order FIR filter. Fig. 5 compared the model estimation results using 50th/400th order FIR filters and 8th order ORTFIR filter. From Fig. 5, it is observed that with 50th order FIR filter, the essential dynamics of the physical system can hardly be catched. With 400th order FIR filter, even though the physical system can be correctly approximated, the estimated model has evident variation, especially in the high frequency range. On the other hand, with 8th order ORTFIR filter, the physical system can be perfectly approximated, no visible variation of the estimated model was found in a wider frequency range.

\begin{tabular}{|c|c|c|c|c|c|c|c|c|}
\hline Order & 1000 & 500 & 400 & 300 & 200 & 100 & 50 & 8 \\
\hline Filter Type & FIR & FIR & FIR & FIR & FIR & FIR & FIR & ORTFIR \\
\hline Variance of Simulation Error & 42.53 & 36.46 & 36.18 & 36.70 & 40.28 & 52.91 & 70.10 & 13.18 \\
\hline
\end{tabular}

Table 1. Model Estimation Results Using FIR Fiter and ORTFIR Filter.

\section{Modal parameters estimation-frequency PolyMAX identification}

A rather general frequency-domain identification method using the standard least squares estimator algorithm is introduced and applied to extract the modal characteristics of a dynamic system from a set of measured data. Consider a set of noisy complex Frequency Response Functions (FRF) measurement data, $G\left(\omega_{j}\right),(j=1, \cdots, N)$. The approximation of the data by a model $P(\omega)$ is addressed by considering the following additive error,

$$
E\left(\omega_{j}\right)=G\left(\omega_{j}\right)-P\left(\omega_{j}\right) \quad j=1, \cdots, N
$$




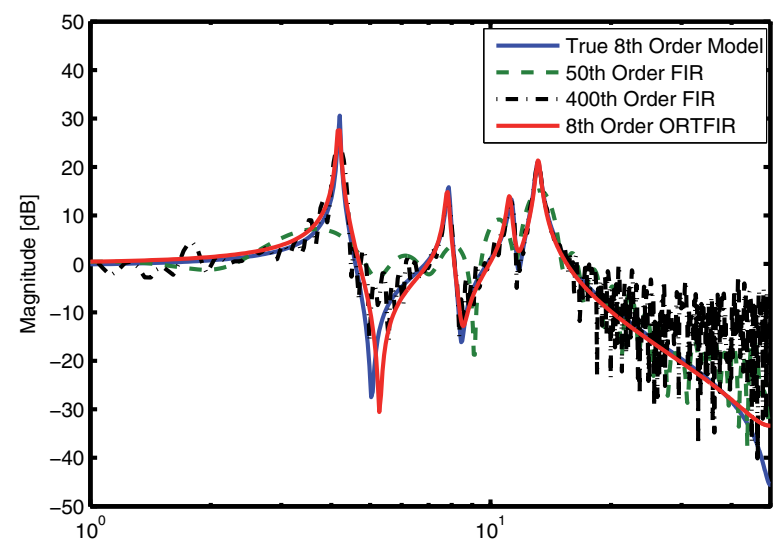

Fig. 5. Comparison of the Model Estimation Using the FIR and ORTFIR Filters.

Then, it is assumed that the model, $P(\omega)$, can be represented by a right polynomial fraction matrix given by,

$$
P(\omega)=[B(\omega)][A(\omega)]^{-1}
$$

where $P(\omega) \in \mathcal{C}^{p \times m}$ is the FRF matrix with $p$ outputs and $m$ inputs, $B(\omega) \in \mathcal{C}^{p \times m}$ is the numerator matrix polynomial, and $A(\omega) \in \mathcal{C}^{m \times m}$ is the denominator matrix polynomial.

The matrix polynomial $B(\omega)$ is parameterized by

$$
B(\omega)=\sum_{k=0}^{n_{b}} B_{k} \xi_{k}(\omega)
$$

where $B_{k} \in \mathcal{R}^{p \times m}$, and $n_{b}$ is the number of non-zero matrix coefficients in $B(\omega)$, or the order of $B(\omega) . \xi_{k}(\omega)$ are the polynomial basis functions. For continuous time model, $\xi_{k}(\omega)=-i \omega_{k}$. For discrete time model, $\xi_{k}(\omega)=e^{-i \omega_{k} T}$ ( $T$ is the sampling time).

The matrix polynomial $A(\omega)$ is parameterized by

$$
A(\omega)=\sum_{k=0}^{n_{a}} A_{k} \xi_{k}(\omega)
$$

where $A_{k} \in \mathcal{R}^{m \times m}$, and $n_{a}$ is the number of non-zero matrix coefficients in $A(\omega)$.

Assuming that the coefficients of the denominator, $A(\omega)$, are $\left[A_{0}, A_{1}, \cdots, A_{n_{a}}\right]$, then, a constraint, $A_{0}=I_{m}$, is set to obtain a stable model to fit the measured frequency-domain data. Here, a constraint, $A_{n_{a}}=I_{m}$, is adopted to extract physical modes from the measured frequency-domain data (Cauberghe et al., 2004).

With, $A_{n_{a}}=I_{m}$, the poles of the estimated model are separated into stable physical poles and unstable mathematical poles, from which a very clean stabilization diagram can be obtained, and the physical modal parameters of the real system can be estimated from a quick evaluation of the generated stabilization diagram (Cauberghe et al., 2005).

The stabilization diagram assumes an increasing model order (number of poles noted in the left ordinate axis), and it indicates where on the frequency axis the poles are located. As a 
rule, unstable poles are not considered in the plot. Physical poles will appear as stable poles, independent of the number of the assumed model order. On the other hand, mathematical poles that intent to model the noise embedded in the data, will change with the assumed model order.

As an example, the 4-DOF lumped system used in the Section 3 is applied in this section to demonstrate the PolyMAX identification method. Fig. 6 (a) depicts the stabilization diagram for the 4-DOF lumped system where four physical modes can be easily appreciated with the parameter constraint of $A_{n_{a}}=I_{1}$. The estimation results can be easily extracted through the access of the stabilization diagram, and they are shown in Table 2. However, with in $A_{n_{0}}=I_{1}$, the physical modes are difficult to extract from the stabilization diagram, because with this parameter constraint, all the mathematical poles are also estimated as the stable poles. This phenomenon is illustrated in Fig. 6 (b). In Fig. 6 (a) and (b), the solid curve indicates the frequency response function (FRF) estimate from the input and output time domain data, the dotted curve indicates the estimated model with the highest order 50 . The right ordinate axis is the magnitude in $\mathrm{dB}$, and which is used to present in the frequency domain the magnitude of the FRF and the estimated 50th order model. The markers indicate different damping values of each estimated stable poles displayed in the stabilization diagram. The detailed meanings of these damping markers are presented in Table 3.

In Table 2, the second column indicates the frequency and damping of the true modes. The third column presents the estimated frequency and damping of the true modes using the proposed PolyMAX identification method and stabilization diagram. Comparing the estimated modes and real modes (calculated from the mathematical equation of motion of 4-DOF lumped system) in Table 2 , it is obvious to see that the frequency, $f_{i}$, and damping, $\zeta_{i}(i=1,2,3,4)$, of these four physical modes are estimated consistently.

\begin{tabular}{|c|c|c|}
\hline & Real Modes & Identified Modes \\
\hline$f_{1}(\mathrm{~Hz})$ & 4.1866 & 4.1868 \\
$f_{2}(\mathrm{~Hz})$ & 7.8648 & 7.8630 \\
$f_{3}(\mathrm{~Hz})$ & 11.3191 & 11.3303 \\
$f_{4}(\mathrm{~Hz})$ & 13.1320 & 13.1320 \\
\hline$\zeta_{1}(\%)$ & 0.5261 & 0.5622 \\
$\zeta_{2}(\%)$ & 0.9883 & 1.1035 \\
$\zeta_{3}(\%)$ & 1.4224 & 1.4836 \\
$\zeta_{4}(\%)$ & 1.6502 & 1.6333 \\
\hline
\end{tabular}

Table 2. Comparison Between the Identified Modes and Real Modes.

\begin{tabular}{|c|c|}
\hline Range of Damping Ratio & Marker Sign \\
\hline $0<\zeta<0.1 \%$ & + \\
$0.1 \%<\zeta<1 \%$ & $\times$ \\
$1 \%<\zeta<2 \%$ & $*$ \\
$2 \%<\zeta<4 \%$ & $\diamond$ \\
$4 \%<\zeta<6 \%$ & $\nabla$ \\
$6 \%<\zeta$ & $\triangle$ \\
\hline
\end{tabular}

Table 3. Damping Markers in the Stabilization Diagram 


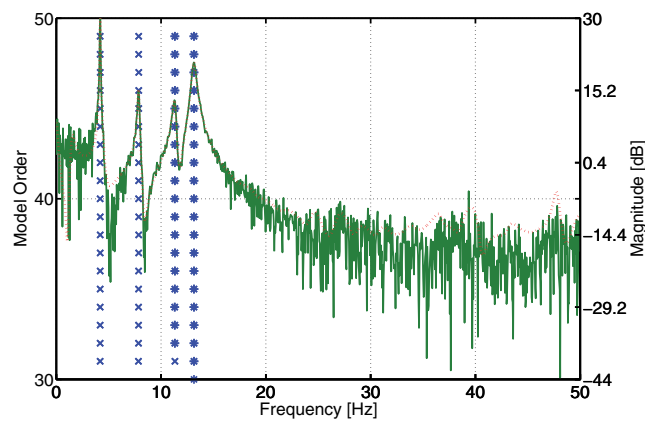

(a) Stabilization Diagram with $A_{n_{a}}=I_{1}$

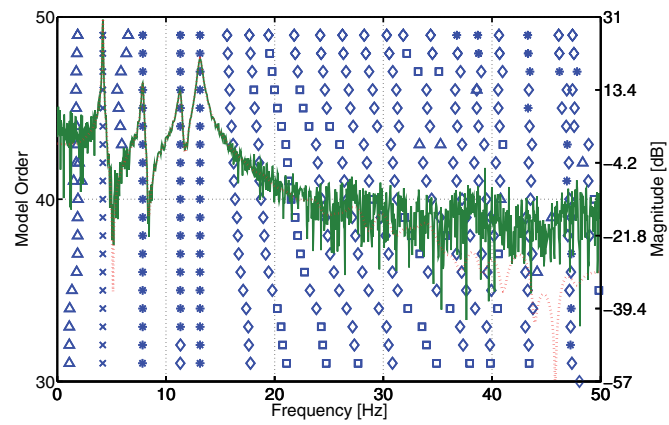

(b) Stabilization Diagram with $A_{n_{0}}=I_{1}$

Fig. 6. Illustration of Stabilization Diagram Using PolyMAX Identification Method (for the Meaning of the Markers, See Table 3).

\section{Recursive least square adaptive algorithm}

The adaptive algorithm to be implemented is the Recursive Least Square (RLS) algorithm (Haykin, 2002). Given the input and output data can be written in regressor form:

$$
y(t)=\phi^{T}(t) \theta+e(t), \theta=\left[\beta_{0}, \beta_{1}, \ldots, \beta_{L-1}\right]^{T}
$$

where $\phi^{T}(t)=\left[u_{0}^{T}(t), \ldots, u_{L-1}^{T}(t)\right]$ is the available input data vector, $\theta$ is the parameter vector to be estimated of the ORTFIR feedforward controller, and $e(t)$ is the residue error. The parameters, $\theta$, can be identified with the available input-output data up to time $t$ by a standard RLS algorithm. It is well known that the RLS algorithm at the steady-state operation exhibits a windup problem if the forgetting factor remains constant, which will deteriorate the estimation results. As a result, a variable forgetting factor (Park, 1991), is sought to prevent this problem from occurring. The parameters, $\theta$, can be estimated by the RLS algorithm using a variable forgetting factor through a two steps approach at each sample time:

1. Compute the gain vector, $k(t)$, and the parameters, $\hat{\theta}(t)$, at the current sample time as:

$$
\hat{\theta}(t)=\hat{\theta}(t-1)+k(t) \xi^{T}(t)
$$




$$
\begin{gathered}
\xi(t)=y(t)-\hat{\theta}^{T}(t-1) \phi(t) \\
k(t)=\frac{P(t-1) \phi(t)}{\lambda_{1}(t)+\phi^{T}(t) P(t-1) \phi(t)}
\end{gathered}
$$

2. Update the inverse correlation matrix, $P(t)$, and the forgetting factor, $\lambda(t)$ :

$$
\begin{gathered}
P(t)=\lambda(t)^{-1}\left[1-k(t) \phi^{T}(t)\right] P(t-1) \\
\lambda(t)=\lambda_{\text {min }}+\left(1-\lambda_{\text {min }}\right) \cdot 2^{-L(t)} \\
L(t)=\quad \operatorname{round}\left(\rho \cdot \xi(t)^{2}\right)
\end{gathered}
$$

where $\rho$ is a design parameter which controls the change rate and the width of anity zone, $\xi(t)$ is the estimation error which is calculated via Eq. (20). $L(t)$ is defines as the nearest integer of $\rho \cdot \xi(t)^{2}$ at each instant time step. $\lambda_{\text {min }}$ defines the lower bound of the $\lambda$.

In Eq. (23), it is shown that when the estimation error, $\xi(t)$ and $L(t)$ is small, $2^{-L(t)} \longrightarrow 1$, and $\lambda(t) \longrightarrow 1$ at an exponential rate, and this rate is controller by $\rho$. When $\xi(t)$ increases to infinity, $\lambda$ reaches its minimum value. The RLS minimization is posted as:

$$
J(t)=\sum_{i=1}^{t} \lambda(i)^{t-i}\left[y(i)-\hat{\theta}(t)^{T} \phi(i)\right]^{2}
$$

By choosing the variable forgetting factor indicated in Eq. (23), the fast decrease of the inverse correlation matrix, $P(t)$, can be avoided at the beginning of the estimation. In general this will result in an accelerated convergence by maintaining a high adaptation at the beginning of the estimation when the parameters, $\theta$, are still far from the optimal value.

\section{Application to closed loop F/A-18 AAW linear model}

\subsection{Linear aeroelastic solver formulation approach}

A unified aeroelastic formulation to take into account the influence of aeroelastic effects on the flight dynamic behavior of the whole aircraft has been developed in (Baldelli et al., 2006).

A general formulation of a flexible aircraft with respect to a body-fixed reference system driven by aerodynamic, thrust, and gravity $(g)$ forces and moments can be defined as:

$$
\begin{aligned}
m\left[\dot{V}_{b}+\Omega_{b} \times V_{b}-R_{b g}(E)[0,0, g]^{T}\right] & =F_{A}+F_{\delta}+F_{T}+\Delta_{F} \\
J \dot{\Omega}_{b}+\Omega_{b} \times J \Omega_{b} & =M_{A}+M_{\delta}+M_{T}+\Delta_{M}
\end{aligned}
$$

where, $m$ and $J$ are the air vehicle mass and inertia tensor, and $R_{b g}(E)$ is the rotation mapping from inertial to body-axes, $(E=[\phi, \theta, \psi])$.

Eq. (25) is driven by the forces and moments on its right hand side, where $F_{A}$ and $M_{A}$ are the external aerodynamic forces and moments on the air vehicle. $F_{A}$ and $M_{A}$ are a function of the aerodynamic flight states $(V \alpha, \beta, E, \ldots$, etc. $)$, Mach number, body angular rates $\left(\Omega_{b}\right)$, and control surface deflections and are usually obtained by wind-tunnel or flight tests. In either case, the quasi-steady influence of the deformed air-vehicle is included by considering flexible-to-rigid ratios or Parameter Identification (PID) techniques, (Morelli, 1995; Morelli \& Klein, 1997). $F_{\delta}$ and $M_{\delta}$ are the aerodynamic forces and moments from the control surfaces 
commanded by the flight control system and pilot inputs while $F_{T}$ and $M_{T}$ includes the thrust loads.

In addition, $\Delta_{F}$ and $\Delta_{M}$ are the aeroelastic incremental loads due to the structural deformation. Usually, these loads are assumed to be quasi-statics and can be computed by a static aeroelastic analysis. However, this quasi-static assumption may not be sufficient for a highly reconfigurable and flexible aircraft like the new generation of Morphing UAV, HALEs, etc., where the interaction between the dynamic structural deformation due to unsteady flow and rigid body motion can play an important role.

During the integration process, the aeroelastic equations of motion underwent two similarity transformation steps, so the generalized coordinates related with the six rigid-body modes originally defined in principle axes are mapped into the airframe states (stability-axes definition). Specifically, for symmetric maneuvers the transformation matrix, $\left[T_{A}\right]_{\text {long }}$ (Baldelli et al., 2006), reads as:

$$
\left\{\begin{array}{c}
T_{x} \\
T_{z} \\
R_{y} \\
\dot{T}_{x} \\
\dot{T}_{z} \\
\dot{R}_{y}
\end{array}\right\}=\underbrace{\left[\begin{array}{cccccc}
-1 & 0 & 0 & 0 & 0 & 0 \\
0 & 0 & 1 & 0 & 0 & 0 \\
0 & 0 & 0 & 0 & 1 & 0 \\
0 & -1 & 0 & 0 & 0 & 0 \\
0 & 0 & 0 & -V & V & 0 \\
0 & 0 & 0 & 0 & 0 & 1
\end{array}\right]}_{\left[T_{A}\right]_{\text {long }}}\left\{\begin{array}{l}
x \\
u \\
h \\
\alpha \\
\theta \\
q
\end{array}\right\}
$$

For an anti-symmetric maneuver, $\left[T_{A}\right]_{\text {lat }}$ is,

$$
\left\{\begin{array}{c}
T_{y} \\
R_{x} \\
R_{z} \\
\dot{T}_{y} \\
\dot{R}_{x} \\
\dot{R}_{z}
\end{array}\right\}=\underbrace{\left[\begin{array}{cccccc}
1 & 0 & 0 & 0 & 0 & 0 \\
0 & 0 & 0 & 0 & -1 & 0 \\
0 & 0 & 0 & 0 & 0 & -1 \\
0 & V & 0 & 0 & 0 & V \\
0 & 0 & -1 & 0 & 0 & 0 \\
0 & 0 & 0 & -1 & 0 & 0
\end{array}\right]}_{\left[T_{A}\right]_{\text {lat }}}\left\{\begin{array}{l}
y \\
\beta \\
p \\
r \\
\phi \\
\psi
\end{array}\right\}
$$

For an asymmetric maneuver, the matrix $\left[T_{A}\right] \in \mathbf{R}^{12 \times 12}$ will be composed by the proper allocation of the elements that form the rows and columns of the $\left[T_{A}\right]_{\text {long }}$ and $\left[T_{A}\right]_{\text {lat }}$ matrices. In this new coordinate system, the linear aeroelastic Equations of Motion (EoM) are:

$$
\begin{aligned}
& \left\{\left[\begin{array}{cc}
M_{r r} & 0 \\
0 & M_{e e}
\end{array}\right] s^{2}+\left[\begin{array}{cc}
C_{r r} & 0 \\
0 & C_{e e}
\end{array}\right] s+\left[\begin{array}{cc}
K_{r r} & 0 \\
0 & K_{e e}
\end{array}\right]\right\}\left\{\begin{array}{l}
\xi_{a s} \\
\eta_{e}
\end{array}\right\}= \\
& q_{\infty}\left\{\left[\begin{array}{ll}
Q_{r r}(s) & Q_{r e}(s) \\
Q_{e r}(s) & Q_{e e}(s)
\end{array}\right]\left\{\begin{array}{c}
\xi_{a s} \\
\eta_{e}
\end{array}\right\}+\left[\begin{array}{l}
Q_{r \delta}(s) \\
Q_{e \delta}(s)
\end{array}\right] \delta_{u}+\left[\begin{array}{cc}
1 & Q_{r G}(s) \\
V & Q_{e G}(s)
\end{array}\right] w_{G}\right\}
\end{aligned}
$$

where $w_{G}$ is the gust input; the elastic generalized coordinates, $\eta_{e}$, input, and $\delta_{u}$, vectors are,

$$
\begin{aligned}
\eta_{e}^{T} & =\left[\eta_{e_{1}}, \ldots, \eta_{e_{N e}}\right]^{T} \\
\delta_{u}^{T} & =\left[\delta_{\text {elev }}, \delta_{\text {ail }}, \delta_{\text {rud }}, \ldots\right]^{T}
\end{aligned}
$$

It should be noted that the equations are only coupled via external forces and moments. In addition, after the transformation is applied the generalized mass matrix of the finite element model, it is no longer necessarily diagonal. In fact, the sub-matrix, $M_{r r}$, associated with the rigid body modes is identical to the mass matrix in the flight dynamics equation; i.e. the 
off-diagonal terms contain the products of inertia,

$$
M_{r r}=\operatorname{diag}\left(m I_{3}, J\right)
$$

Usually, the aerodynamic force coefficient matrix, $Q(s)$, is approximated using the Rational Function Approximation (RFA) approach as

$$
Q(s)=\left[A_{0}\right]+\left[A_{1}\right] \frac{L}{V} s+\left[A_{2}\right] \frac{L^{2}}{V^{2}} s^{2}+[D]\left(s I-\frac{V}{L}[R]\right)^{-1}[E] s
$$

where the $\left[A_{i}\right], i=0,1,2,[D]$ and $[E]$ matrices are column partitioned as,

$$
\left[A_{i}\right]=\left[A_{r} A_{e} A_{\delta}\right]
$$

where $i=r, e, \delta$ are the airframe, elastic and control related states.

In this formulation, the $\left[A_{i}\right]$ coefficient matrices represent the quasi-steady aerodynamic forces, and the remanent terms are used to model the flow unsteadiness by Padé approximation.

Using the Minimum-State approach during the RFA implemented in the ZAERO/ASE module (Karpel, 1992), and due to the performed similarity transformation the aero-lag terms are computed as,

$$
\begin{aligned}
\left\{\dot{x}_{L}\right\} & =\frac{V}{L}[R]\left\{x_{L}\right\}+\left[\begin{array}{llll}
E_{L *} & E_{L r} & E_{L e} & E_{L \delta}
\end{array}\right]\left\{\begin{array}{c}
\xi_{a s} \\
\dot{\xi}_{a s} \\
\dot{\eta}_{e} \\
\delta_{\dot{u}}
\end{array}\right\} \\
z_{L} & =[D]\left\{x_{L}\right\}
\end{aligned}
$$

By including Eqs. (30) and (32) into Eq. (28), the aeroelastic EoM can now be easily partitioned in accordance with the airframe degrees of freedom, elastic dynamics, aerodynamic lag terms, a set of control inputs and gust perturbation as:

$$
\begin{aligned}
\left\{\begin{array}{c}
\dot{\xi}_{a s} \\
\ddot{\xi}_{a s} \\
\dot{\eta}_{e} \\
\ddot{\eta}_{e} \\
\dot{x}_{L}
\end{array}\right\}= & {\left[\begin{array}{cc|ccc}
A_{\xi_{a s}} & A_{\dot{\xi}_{a s}} & 0 & 0 & 0 \\
A_{r r_{0}} & A_{r r_{1}} & A_{r e_{0}} & A_{r e_{1}} & A_{r L} \\
\hline 0 & 0 & 0 & I & 0 \\
A_{e r_{0}} & A_{e r_{1}} & A_{e e_{0}} & A_{e e_{1}} & A_{e L} \\
E_{L *} & E_{L r} & 0 & E_{L e} & V
\end{array}\right]\left\{\begin{array}{c}
\xi_{a s} \\
\dot{\xi}_{a s} \\
\eta_{e} \\
\dot{\eta}_{e} \\
x_{L}
\end{array}\right\} } \\
& +\left[\begin{array}{ccc}
0 & 0 & 0 \\
B_{r_{0}} & B_{r_{1}} & B_{r_{2}} \\
\hline 0 & 0 & 0 \\
B_{e_{0}} & B_{e_{1}} & B_{e_{2}} \\
0 & E_{L \delta} & 0
\end{array}\right]\left\{\begin{array}{c}
\delta_{u} \\
\delta_{\dot{u}} \\
\delta_{\ddot{u}}
\end{array}\right\}+\left[\begin{array}{cc}
0 \\
B_{r_{w 1}} & B_{r_{w 22}} \\
\hline 0 & 0 \\
B_{e_{w 1}} & B_{e_{w 2}} \\
0 & E_{r G}
\end{array}\right]\left\{\begin{array}{l}
w_{G} \\
\dot{w}_{G}
\end{array}\right\}
\end{aligned}
$$

where $A_{\tilde{\xi}_{a s}}, A_{\dot{\xi}_{a s}}$ and $E_{L *}$ are coupling matrices due to the similarity transformation executed. Now, the aeroelastic incremental loads, $\Delta_{F}$ and $\Delta_{M}$, should be implemented in a way to allow a seamless integration between the nonlinear flight dynamics and the linear aeroelastic EoMs. In fact, this can be easily achieved in accordance with the partitions showed in Eq. (34) between rigid, elastic and aerodynamic lag dynamics. Hence, the aeroelastic incremental 
loads are computed similarly to the approximation given by Eq. (30),

$$
\left[\begin{array}{c}
\Delta_{F} \\
\Delta_{M}
\end{array}\right]=q_{\infty}\left\{A_{0_{r e}} \bar{\eta}_{e}+A_{1_{r e}} \frac{L}{V} \dot{\eta}_{e}+A_{2_{r e}} \frac{L^{2}}{V^{2}} \ddot{\eta}_{e}+D_{r e} x_{L e}\right\}
$$

Clearly to implement this algebraic equation, the generalized coordinate, $\bar{\eta}_{e}=\eta_{e}-\eta_{e_{0}}$, its rate, $\dot{\eta}_{e}$, and acceleration, $\ddot{\eta}_{e}$, vectors as well as the aerodynamic lag terms related with the elastic modes, $x_{L_{e}}$, must be estimated at each time iteration.

Note that the Minimum-State method is formulated to only use a single set of lag states, $x_{L}$, in Eq. (32). Therefore, the following augmented equation is devised to decouple the generalized coordinates' aero lag terms from the airframe states, $\xi_{a s}$ and $\dot{\xi}_{a s}$, related ones,

$$
\begin{aligned}
& \left\{\begin{array}{c}
\dot{x}_{L_{a s}} \\
\dot{x}_{L_{e}}
\end{array}\right\}=\left[\begin{array}{cc}
V & 0 \\
0 & \frac{V}{L} R
\end{array}\right]\left\{\begin{array}{c}
x_{L_{a s}} \\
x_{L_{e}}
\end{array}\right\}+\left[\begin{array}{cccc}
E_{L *} & E_{L r} & 0 & E_{L e} \\
0 & 0 & 0 & E_{L e}
\end{array}\right]\left\{\begin{array}{c}
\xi_{a s} \\
\xi_{a s} \\
\eta_{e} \\
\dot{\eta}_{e}
\end{array}\right\} \\
& +\left[\begin{array}{lll}
0 & 0.5 E_{L \delta} & 0 \\
0 & 0.5 E_{L \delta} & 0
\end{array}\right]\left\{\begin{array}{l}
\delta_{u} \\
\delta_{\dot{u}} \\
\delta_{\ddot{u}}
\end{array}\right\}+\left[\begin{array}{ll}
0 & 0.5 E_{r G} \\
0 & 0.5 E_{r G}
\end{array}\right]\left\{\begin{array}{l}
w_{G} \\
\dot{w}_{G}
\end{array}\right\} \\
& \left\{\begin{array}{c}
z_{L_{a s}} \\
z_{L_{e}}
\end{array}\right\}=\left[\begin{array}{ll}
D & D
\end{array}\right]\left\{\begin{array}{c}
x_{L_{a s}} \\
x_{L_{e}}
\end{array}\right\}
\end{aligned}
$$

In this way, only elastic lag terms are considered to avoid any possible coupling with the rigid-body airframe related states (i.e. $\xi_{a s}$ and $\xi_{a s}$ ). Now, the following differential equation is obtained combining the lower partition of Eq. (34) with the new devised Eq. (36).

$$
\begin{aligned}
& \underbrace{\left\{\begin{array}{c}
\dot{\eta}_{e} \\
\ddot{\eta}_{e} \\
\dot{x}_{L_{a s}} \\
\dot{x}_{L_{e}}
\end{array}\right\}}_{\dot{x}_{e}}=\underbrace{\left[\begin{array}{cc|cc}
0 & I & 0 & 0 \\
A_{e e_{0}} & A_{e e_{1}} & A_{e L} & A_{e L} \\
\hline 0 & E_{L e} & \frac{V}{\bar{c}} R & 0 \\
0 & E_{L e} & 0 & \frac{V}{\bar{c}} R
\end{array}\right]}_{A} \underbrace{\left\{\begin{array}{c}
\eta_{e} \\
\dot{\eta}_{e} \\
x_{L_{a s}} \\
x_{L_{e}}
\end{array}\right\}}_{x_{e}}+\underbrace{\left[\begin{array}{ccc}
0 & 0 & 0 \\
B_{e_{0}} & B_{e_{1}} & B_{e_{2}} \\
0 & 0.5 E_{L \delta} & 0 \\
0 & 0.5 E_{L \delta} & 0
\end{array}\right]}_{B_{1}} \underbrace{\left\{\begin{array}{c}
\delta_{u} \\
\delta_{\dot{u}} \\
\delta_{\ddot{u}}
\end{array}\right\}}_{\delta_{U}} \\
& +\underbrace{\left[\begin{array}{cc}
0 & 0 \\
A_{e r_{0}} & A_{e r_{1}} \\
E_{L *} & E_{L r} \\
0 & 0
\end{array}\right]}_{B_{2}}\{\underbrace{\left\{\begin{array}{l}
\delta_{\xi_{a s}} \\
\delta_{\dot{\xi}_{a s}}
\end{array}\right\}}_{\delta_{\xi}}+\underbrace{\left[\begin{array}{cc}
0 & 0 \\
\frac{B_{e w 1}}{B_{e w 2}} \\
0 & 0.5 E_{r G} \\
0 & 0.5 E_{r G}
\end{array}\right]}_{B_{3}}\{\underbrace{\left\{\begin{array}{c}
w_{G} \\
\dot{w}_{G}
\end{array}\right\}}_{w}
\end{aligned}
$$

where $\delta_{\xi_{a s}}$ and $\delta_{u}$ are defined as the incremental airframe states and inputs (perturbation from trim values),

$$
\begin{aligned}
\delta_{\xi_{a s}} & =\xi_{a s}-\left.\xi_{a s}\right|_{0} \\
\delta_{u} & =u-u_{\mid 0}
\end{aligned}
$$

$\left.\xi_{a s}\right|_{0}$ and $u_{\mid 0}$ being the airframe state and input vectors computed at some specific trim condition ab-initio of the simulation run. Using a short notation form, Eq. (37) can be expressed as,

$$
\dot{x}_{e}=A x_{e}+B_{1} \delta_{U}+B_{2} \delta_{\xi}+B_{3} w
$$


The previous equation is used to estimate the elastic and lag states as a function of the incremental control input, $\left(\delta_{U}^{T}=\left[\delta_{u}^{T}, \delta_{\dot{u}}^{T}, \delta_{\ddot{u}}^{T}\right]^{T}\right)$, and incremental airframe states, $\left(\delta_{\tilde{\xi}}^{T}=\right.$ $\left.\left[\delta_{\tilde{\xi}_{a s}}^{T}, \delta_{\dot{\zeta}_{a s}}^{T}\right]^{T}\right)$ at each time iteration.

The quasi-static elastic deformation, $\eta_{e 0}$, is computed by static residualization of the elastic modes, that is the $\dot{\eta}_{e}=\ddot{\eta}_{e}=x_{L e}=0$ condition needs to be fulfilled. Therefore, the quasi-static elastic influence is estimated from Eq. (40) as:

$$
\dot{x}_{e}=0 \Longrightarrow x_{e}=-A^{-1}\left(B_{1} \delta_{U}+B_{2} \delta_{x} i+B_{3} w\right)
$$

and from $x_{e}$ the quasi-static elastic influence vector, $\eta_{e 0}$, can be recovered.

In summary, the linear aeroelastic solver will be built based on:

1. Algebraic Eq. (35) to compute the incremental aeroelastic loads, $\Delta_{F}$ and $\Delta_{M}$.

2. First-order differential Eq. (40) to compute the generalized coordinates related vectors $\bar{\eta}_{e}$, $\dot{\eta}_{e}$, and $\ddot{\eta}_{e}$, as well as the aerodynamic lag terms related with the elastic modes, $x_{L_{e}}$.

3. Algebraic Eq. (41) to estimate the quasi-static deformation vector $\eta_{e_{0}}$ at that specific flight condition.

Fig. 7 illustrates the interconnection of the F/A-18 AAW 6-DOF Dynamics subsystem and the Incremental Aeroelastic Solver, Control Surface Mixer and Control Command Trans form blocks.

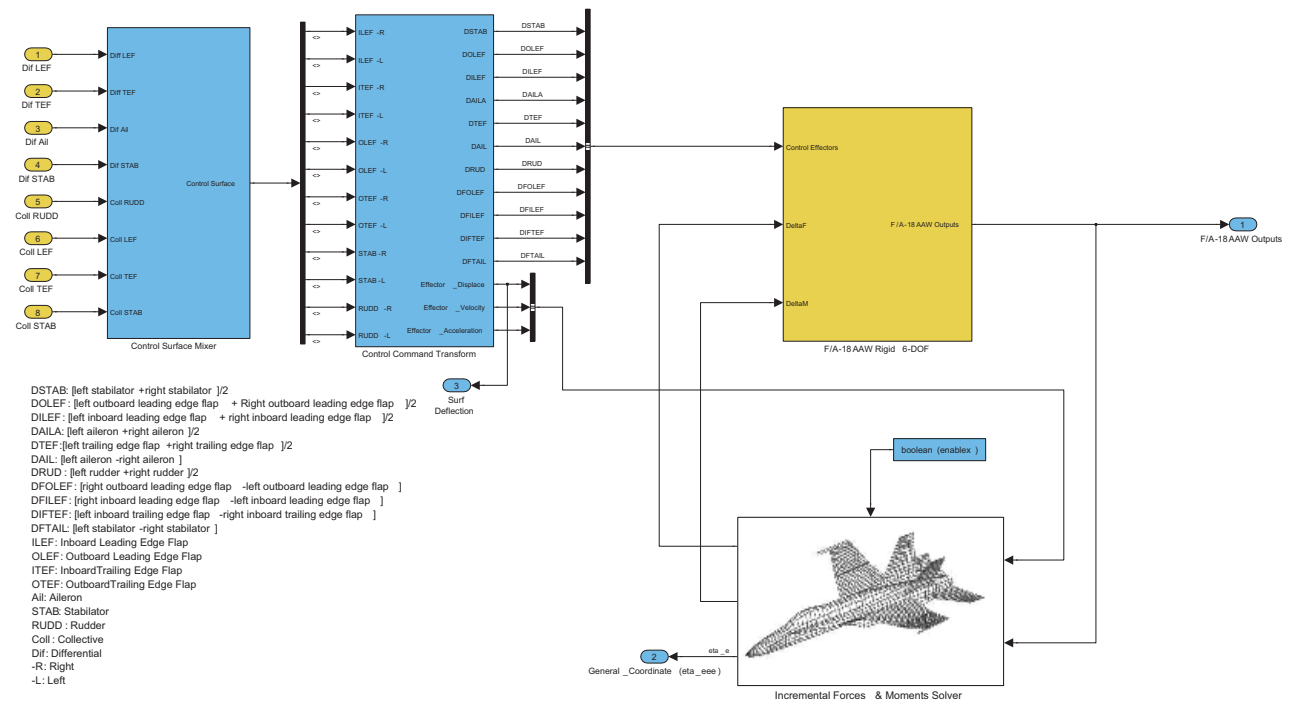

Fig. 7. Addition of the Incremental Aeroelastic Loads Solver to the Nonlinear Rigid-Body 6-DOF Subsystem.

\subsection{Closed loop F/A-18 AAW linear model with gust excitation}

In order to demonstrate the proposed feedforward filter design algorithm, a simplified closed loop F/A-18 AAW linear simulink model with gust excitation is developed/implemented for the evaluation purposes. This high-fidelity aeroelastic model was developed using the following elements: 
- Six-degree-of-freedom solver using Euler angles subsystem.

- The AAW flight control system.

- Actuators and sensors.

- Aerodynamic Forces and Moments subsystem using the set of non-dimensional stability and control derivatives obtained through a set of AAW parameter identification flight tests.

- An incremental aeroelastic load solver including gust excitation generated by the ZAERO software system using rational function approximation techniques.

For continuous vertical gust perturbation, a low pass filter followed by a Dryden vertical velocity shaping filter is used to shape the power of the gust perturbation. The low pass filter is used to obtain the derivative of the gust perturbation. The low pass filter is given as $T_{L P F}(s)=\frac{a}{s+a}$ where $a=200 \pi \mathrm{rad} / \mathrm{s}$ is chosen in the remainder of the section.

The Dryden vertical velocity shaping filter is given as

$$
T_{g}(s)=\sigma_{w_{G}} \frac{\sqrt{3} \tau_{g}^{-1 / 2} s+\tau_{g}^{-3 / 2}}{\left(s+1 / \tau_{g}\right)^{2}}
$$

where $\tau_{g}=L_{g} / V$, and $L_{g}=1750 \mathrm{ft}, V$ is the aircraft body axis velocity; $\sigma_{w_{G}}=100 \mathrm{ft} / \mathrm{s}$.

For a more detailed development of the F/A-18 AAW simulink model with gust excitation, please refer to the NASA SBIR Phase I final report (Zeng \& de Callafon, 2008). The implementation of the adaptive feedforward control algorithm to the linearized F/A-18 AAW simulink model is illustrated in Fig. 8. It should be noted that during the simulation study considered in this paper, the dynamics of airborne LIDAR turbulence sensor has not been considered. We assumed that a perfect gust perturbation can be measured by the airborne LIDAR turbulence sensor, i.e., the sensor dynamics has an ideal constant dynamics of 1 . However, the practical effects of the airborne LIDAR turbulence sensor on the performance of the feedforward controller has to be addressed in the future study.

\subsection{Implementation of the adaptive feedforward control}

The construction of the feedforward controller can be separated into two steps, initialization and the recursive estimation of the filter. In the initialization step, a secondary path transfer function, $G(q)$, is estimated, which is done by performing an experiment using an external signal injected into the left and right trailing edge flaps as the excitation signal, and the error signal, $e(t)$, as the output signal. Since, $\hat{G}(q)$, is only used for filtering purposes, a high order model can be estimated to provide an accurate reconstruction of the filtered input, $\hat{u}_{f}(t)$, via

$$
\hat{u}_{f}(t)=\hat{G}(q) w(t)
$$

as described in Eq. (4).

To facilitate the use of the ORTFIR filter, a set of modal parameters need to be extracted to build the ORTFIR filter, using the frequency domain PolyMAX identification methodology in Section 4. With $\hat{u}_{f}(t)$ given in Eq. (42) and $d(t)=H(q) w(t)$ in place, the modal parameters can be easily estimated using the PolyMAX method. With the signal, $d(t), \hat{u}_{f}(t)$ and the basis function, $B_{i}(q)$, a recursive minimization of the feedforward filter is done via the recursive least squares minimization technique described in Section 5. 


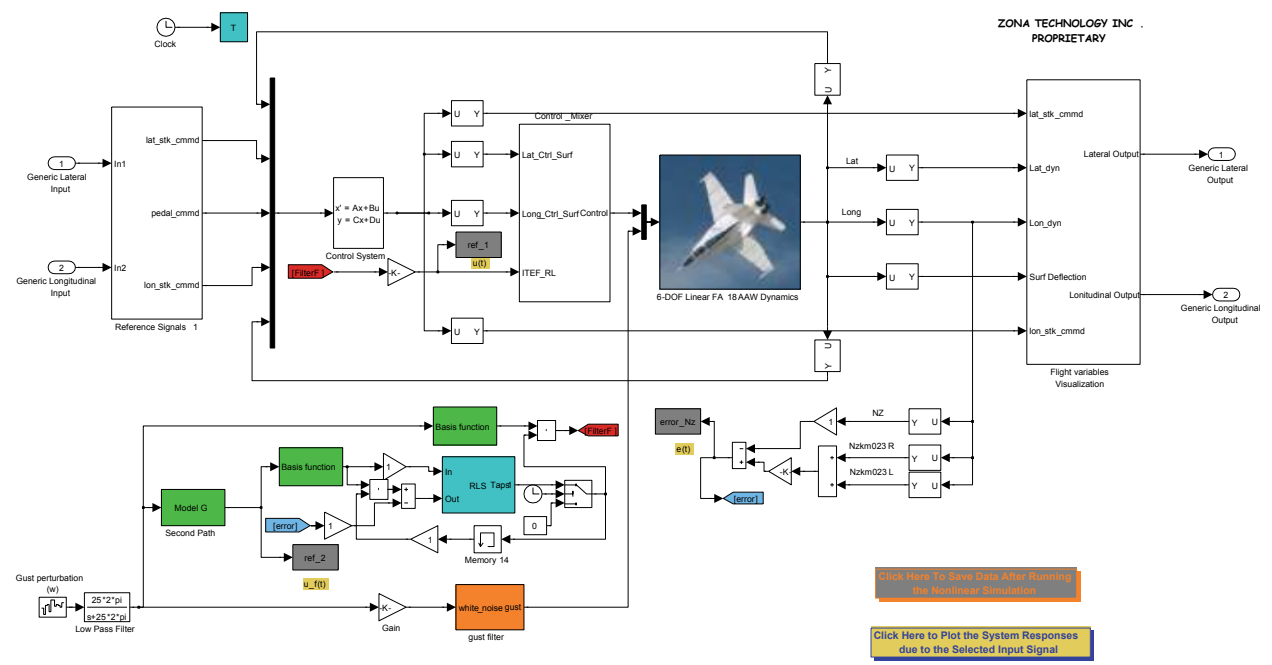

Fig. 8. Closed Loop F/A-18 AAW Linear Simulink Model.

The error signal, $e(t)$, can be selected as the vertical accelerometer reading at the aircraft left/right wing folder positions, i.e., $N z_{k m 023 R}$ or $N z_{k m 023 L}$. An alternative choice could be

$$
e(t)=\left[\frac{N z_{k m 023 R}+N z_{k m 023 L}}{2}-N z_{c g}\right]
$$

In this paper, Eq. (43) is served as a feedback signal for feedforward filter design purpose. The advantage of choosing Eq. (43) is that the rigid body dynamics can be partly removed, and the vertical wing bending is still observed.

Upon initialization of the feedforward controller, a 20th order ORTFIR model, $\hat{G}(q)$, was estimated in order to create the filtered signal, $\hat{u}_{f}(t)$. The amplitude bode plot of the estimated, $\hat{G}(q)$, is shown in Fig. 9.

The modes used to build the orthonormal basis, $B_{i}(q)$ are extracted from the stabilization diagram in Fig. 10. From Fig. 10, five elastic modes can be extracted, and they are shown in Table 4 .

\begin{tabular}{|c|c|c|}
\hline & \multicolumn{2}{|c|}{ PolyMAX Identification } \\
\hline Mode Number & Frequency [Hz] & Damping $\zeta[\%]$ \\
\hline 1 & 5.9246 & 4.5311 \\
\hline 2 & 10.083 & 4.182 \\
\hline 3 & 13.602 & 10.072 \\
\hline 4 & 18.377 & 2.7409 \\
\hline 5 & 21.569 & 2.5183 \\
\hline
\end{tabular}

Table 4. Estimated Modes of Feedforward Filter Using FFT-PolyMAX Method. 

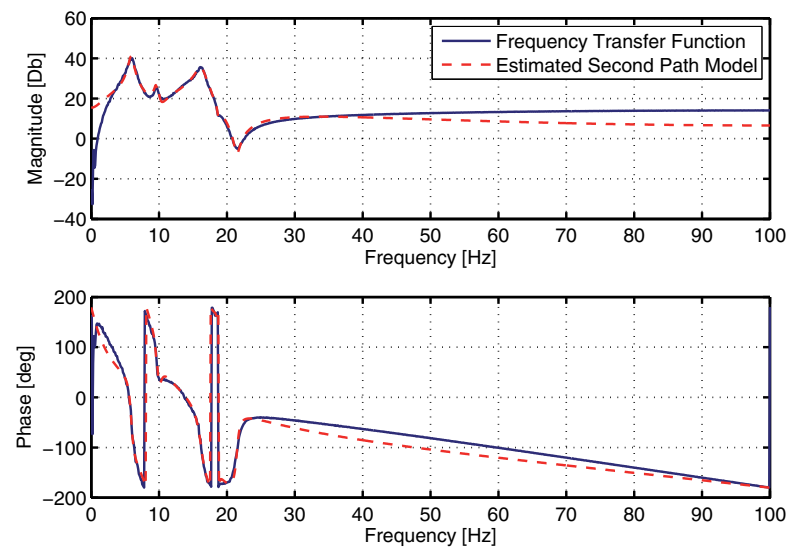

Fig. 9. Bode Plot of the Estimated 20th Order Secondary Path Model, $\hat{G}(q)$.

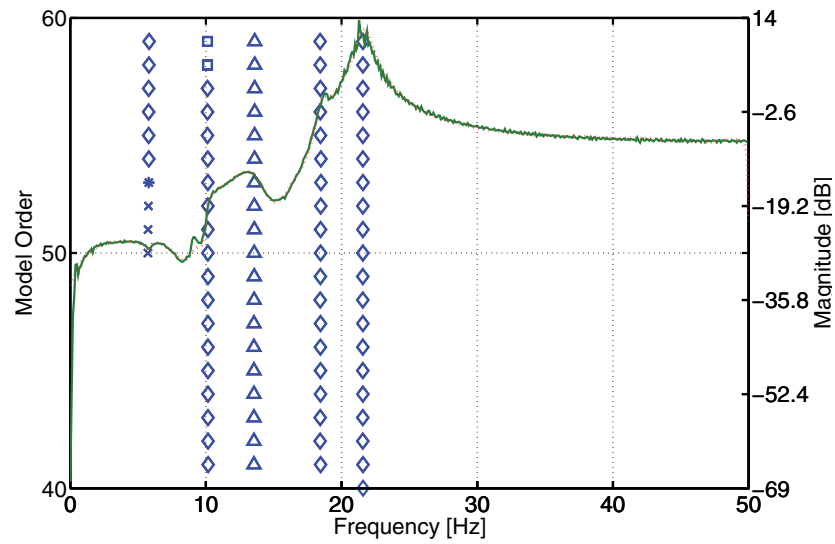

Fig. 10. Stabilization Diagram.

For implementation purposes, only $L=2$ parameters in the ORTFIR filter are estimated. With a 10th order basis, $B_{i}(q)$, this amounts to 20th order ORTFIR filter. To evaluate the performance of the proposed ORTFIR filter for feedforward compensation, a 20th order Finite Impulse Response Filter is also designed to reduce the vertical wing vibration.

For a clear performance comparison between FIR filter and ORTFIR filter, the frequency response of the $N z_{k m 023 R}$, and $N z_{k m 023 L}$ using FIR filter and ORTFIR filter are plotted in Fig. 11 and Fig. 12, respectively. The solid line in Fig. 11 (a) is the auto spectrum of the accelerometer measurement $N z_{k m 023 R}$ without feedforward controller integrated in the system; the dashed line in Fig. 11(a) indicates the auto spectrum of the accelerometer measurement $N z_{k m 023 R}$ with the adaptive feedforward controller using FIR filter added in the system; the dotted line in Fig. 11 shows the auto spectrum of the accelerometer measurement $N z_{k m 023 R}$ with the adaptive feedforward controller using ORTFIR filter added in the system. Fig. 11 (b) is the 


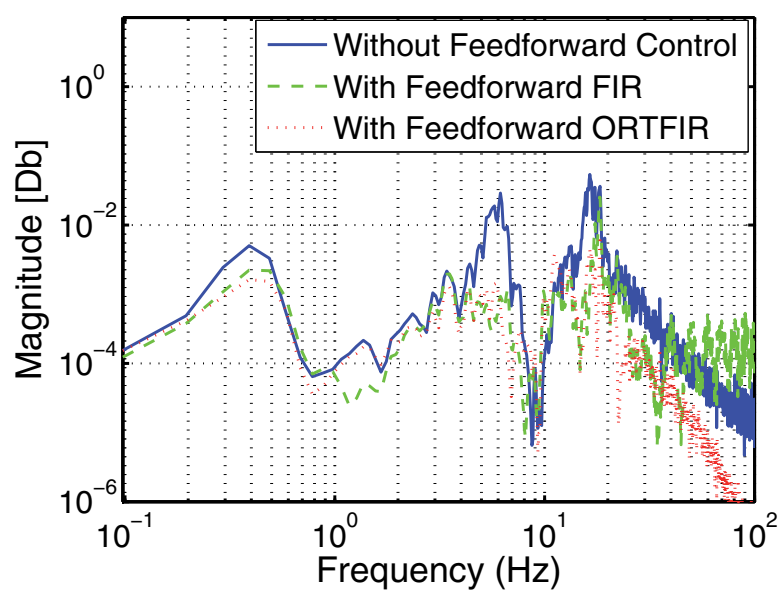

(a) Frequency Spectrum Plot of the $N z_{k m 023 R}$.

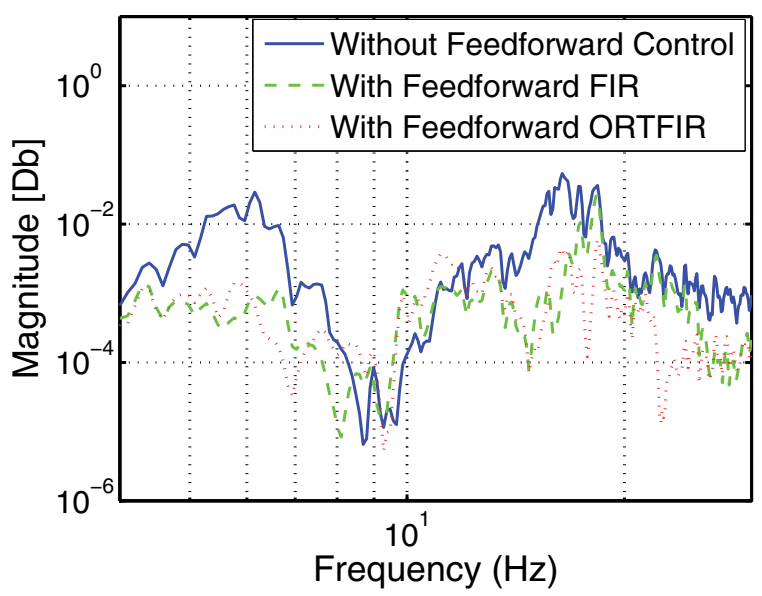

(b) Zoomed Frequency Spectrum Plot of the $N z_{k m 023 R}$.

Fig. 11. Spectral Content Estimates of the $N z_{k m 023 R}$ Without Control (Solid), With Control Using 20th Order FIR Filter (Dashed), and Using 20th Order ORTFIR Filter (Dotted).

zoomed-in plot of Fig. 11 (a) in the frequency range of [4 30] Hz. It is clearly seen that with the ORTFIR filter, a better magnitude reduction of auto spectrum of $N z_{k m 023 R}$ can be obtained in most of the frequency range compared to the FIR filter. Similar performance could also be observed in regards to $N z_{k m 023 L}$, and which is shown in Fig. 12 (a) and (b).

The corresponding time responses are illustrated in Fig. 13 and Fig. 14. Fig. 13 (b) and Fig. 14 (b) are the zoomed-in plots of Fig. 13 (a) and Fig. 14 (a), respectively. These time responses clearly demonstrate that with the adaptive feedforward controller using ORTFIR filter, a better structural vibration reduction can be obtained. From these figures, it is clearly demonstrated 


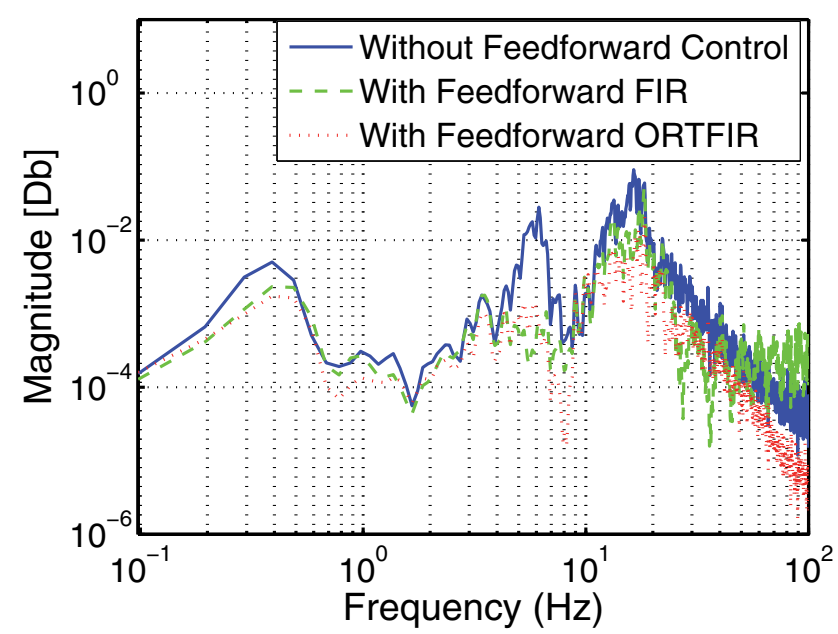

(a) Frequency Spectrum Plot of the $N z_{k m 023 L}$.

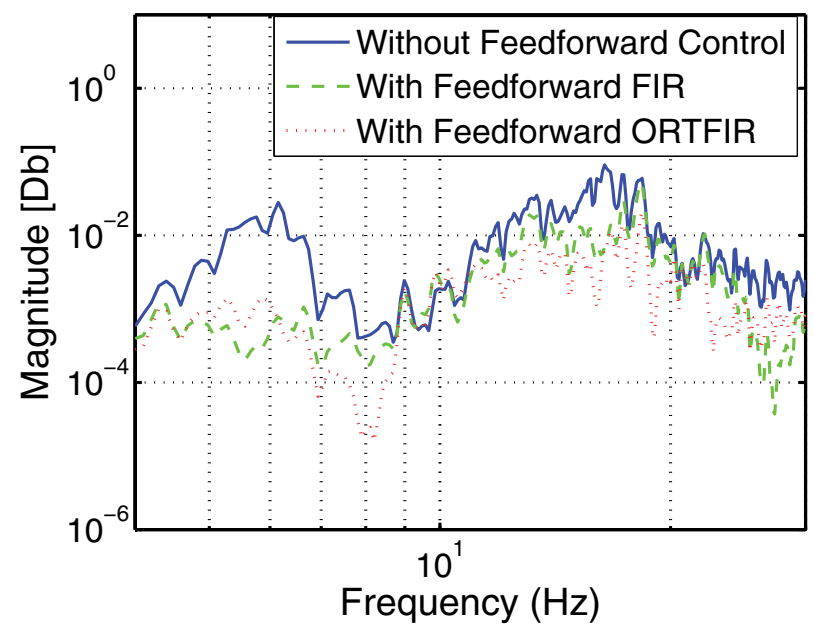

(b) Zoomed Frequency Spectrum Plot of the $N z_{k m 023 L}$.

Fig. 12. Spectral Content Estimates of the $N z_{k m 023 L}$ Without Control (Solid), With Control Using 20th Order FIR Filter (Dashed), and Using 20th Order ORTFIR Filter (Dotted).

that both FIR filter and ORTFIR filter are efficient to reduce the normal acceleration at the left wing folder position and right wing folder position. With the use of the both the ORTFIR filter and FIR filter, the spectral content of the $N z_{k m 023 R}$ and $N z_{k m 023 L}$ have been reduced significantly in the frequency range from $2 \mathrm{~Hz}$ to $20 \mathrm{~Hz}$. However, with the use of the ORTFIR filter, more efficient vibration reduction performances are expected compared to the FIR filter. 


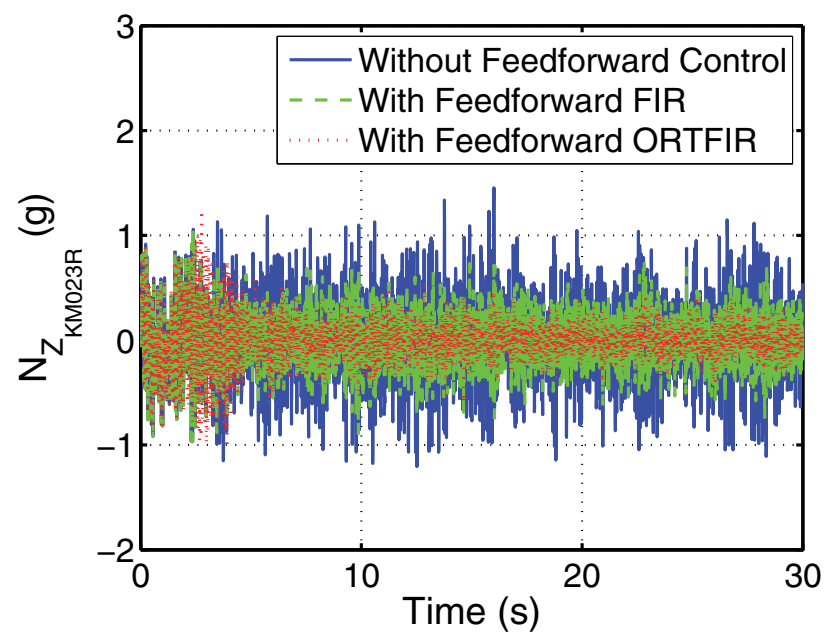

(a) Time Domain Response Plot of the $N z_{k m 023 L}$.

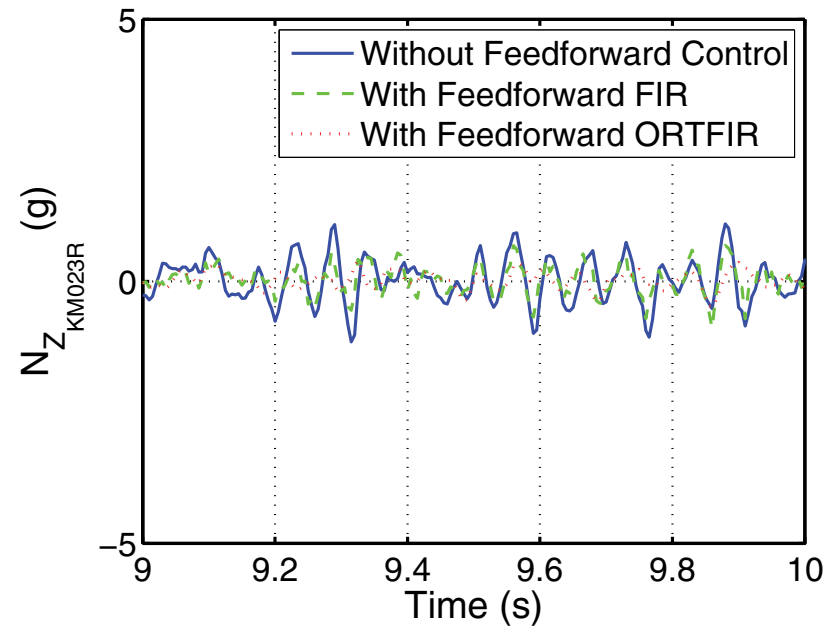

(b) Zoomed Time Domain Response Plot of the $N z_{k m 023 L}$.

Fig. 13. Time domain Response of the $N z_{k m 023 R}$ Without Control (Solid), With Control Using 20th Order FIR Filter (Dashed), and Using 20th Order ORTFIR Filter (Dotted). 


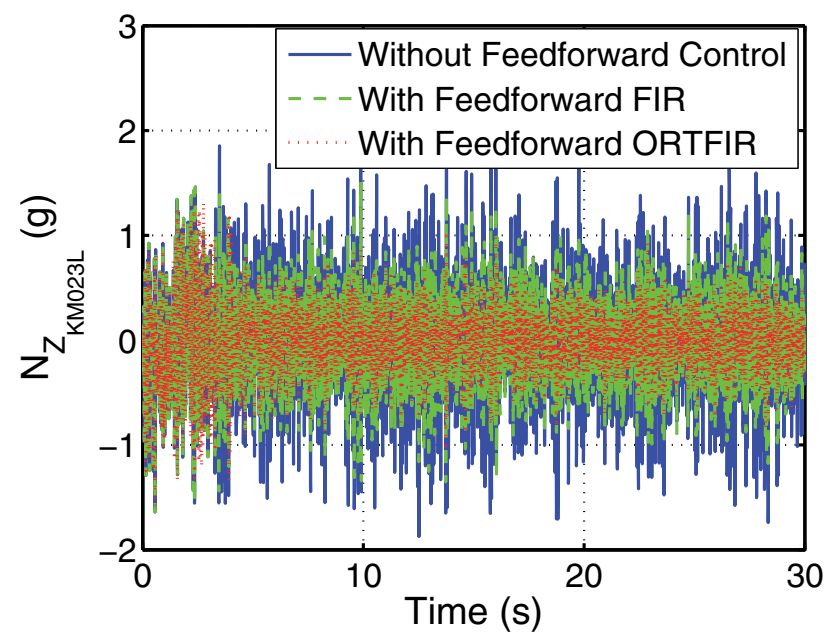

(a) Time Domain Response Plot of the $N z_{k m 023 L}$.

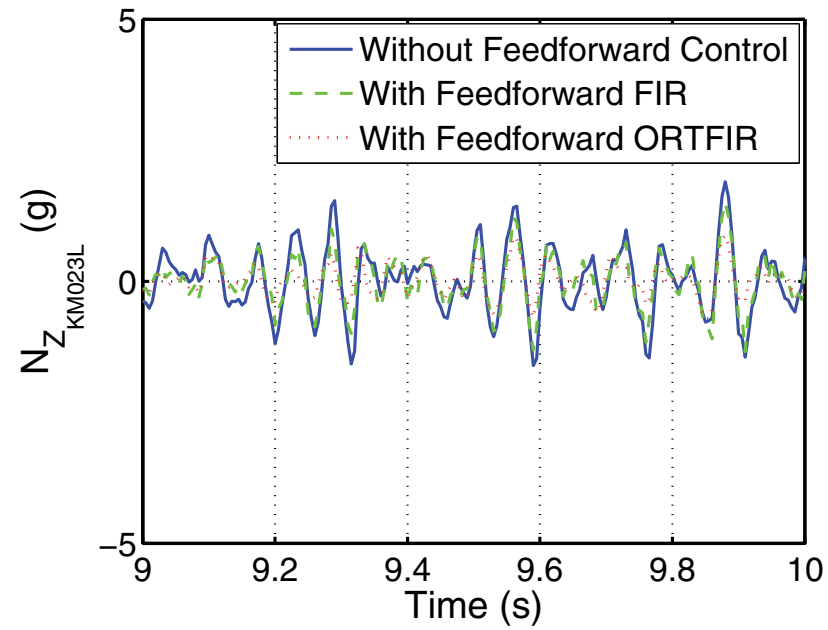

(b) Zoomed Time Domain Response Plot of the $N z_{k m 023 L}$.

Fig. 14. Time Domain Response of the $N z_{k m 023 L}$ Without Control (Solid) and Using 20th Order FIR Filter (Dashed), and With Control Using 20th Order ORTFIR Filter (Dotted). 


\section{Conclusions}

In this chapter, an adaptive feedforward control methodology has been proposed for the active control of gust loads alleviation using an ORTFIR filter. The ORTFIR filter has the same linear parameter structure as a taped delay FIR filter that is favorable for (recursive) estimation. The advantage of using the ORTFIR filter is that it allows the inclusion of prior knowledge of the flexible mode information of the aircraft dynamics in the parametrization of the filter for better accuracy of the feedforward filter.

In addition, by combining the flight dynamics model for rigid body dynamics and an aeroelastic solver for aeroelastic incremental loads to accurately mimic in-flight recorded dynamic behavior of the air vehicle, a unified integration framework that blends flight dynamics and aeroelastic model is developed to facilitate the pre-flight simulation.

The proposed methodology in this chapter is implemented on an F/A-18 AAW aeroelastic model developed with the unified integration framework. The feedforward filter is updated via the recursive least square technique with the variable forgetting factor at each time step. Compared with a traditional FIR filter and evaluated on the basis of the simulation data from the F/A-18 AAW aeroelastic model, it demonstrates that applying the adaptive feedforward controller using the ORTFIR filter yields a better performance of the gust loads alleviation of the aircraft.

\section{References}

Andrighettoni, M. \& Mantegazza, P. (1998). Multi-Input/Multi-output adaptive active flutter suppression for a wing model, Journal of Aircraft 35(3): 462-469.

Baldelli, D. H., Chen, P. C. \& Panza, J. (2006). Unified aeroelastic and flight dynamics formulation via rational function approximations, Journal of Aircraft 43(3): 763-772.

Baldelli, D. H. \& Zeng, J. (2007). Unified nonlinear flight dynamics aeroelastic solver tool, Technical Report SBIR Phase I NNL07AA85P, ZONA Technology Inc.

Baldelli, D. H., Zeng, J., Lind, R. \& Harris, C. (2009). Flutter-prediction tool for flight-test-based aeroelastic parameter-varying models, Journal of Guidance, Control, and Dynamics 32(1): 158-171.

Barker, J. M., Balas, G. J. \& Blue, P. A. (1999). Active flutter suppression via gain-scheduled linear fractional control, Proceedings of the American Control Conference, San Diego, California, pp. 4014-4018.

Cauberghe, B., Guillaume, P., Verboven, P., Parloo, E. \& Vanlanduit, S. (2004). A poly-reference implementation of the maximum likelihood complex frequency-domain estimator and some industrial applications, Proceedings of the 22nd International Modal Analysis Conference, Dearborn, US.

Cauberghe, B., Guillaume, P., Verboven, P., Vanlanduit, S. \& Parloo, E. (2005). On the influence of the parameter constraint on the stability of the poles and the discrimination capabilities of the stabilization diagrams, Mechanical Systems and Signal Processing 19(5): 989-1014.

Eversman, W. \& Roy, I. D. (1996). Active flutter suppresion using MIMO adaptive LMS control, 37th AIAA / ASME / ASCE / AHS / ASC Structures, Structural Dynamics, and Material Conference and Exhibit, Salt Lake, UT. AIAA-1996-1345.

Garrard, W. L. \& Liebst, B. S. (1985). Active flutter suppression using eigenspace and linear quadratic design techniques, Journal of Guidance, Control, and Dynamics 8(3): 304-311.

Haykin, S. (2002). Adaptive Filter Theory, Prentice Hall, Englewood Cliffs, NJ. 
Heuberger, P. S. C., Van Den Hof, P. M. J. \& Bosgra, O. H. (1995). A generalized orthonormal basis for linear dynamical systems, IEEE Transactions on Automatic Control 40(3): 451-465.

Karpel, M. (1992). Size reduction techniques for the determination of efficient aeroservoelastic models, Control and Dynamic Systems 54: 263-295.

Leibst, B. S., Garrard, W. L. \& Farm, J. A. (1988). Design of a multivariable fluter suppression/gust load alleviation system, Journal of Guidance, Control, and Dynamics 11(3): 220-229.

Ljung, L. (1999). System Identification: Theory for the User, Prentice-Hall, Englewood Cliffs, NJ.

Mahesh, J. K., Stone, C. R., Garrard, W. L. \& Dunn, H. J. (1981). Control law synthesis for flutter suppression using linear quadratic control theory, Journal of Guidance, Control, and Dynamics 4(4): 415-422.

Morelli, E. A. (1995). Global nonlinear aerodynamic modeling using multivariate orthogonal functions, Journal of Aircraft 32(2): 270-277.

Morelli, E. A. \& Klein, V. (1997). Accuracy of aerodynamic model parameters estimated from flight test data, Journal Guidance, Control, and Dynamics 20(1): 74-80.

Newsom, J. R. (1979). Control law synthesis for active flutter suppresion using optimal control theory, Journal of Guidance, Control, and Dynamics 2(5): 388-394.

Ninness, B. \& Gustafsson, F. (1997). A unifying construction of orthonormal bases for system identification, IEEE Transactions on Automatic Control 42(4): 515-521.

Park, D. J. (1991). Fast tracking RLS algorithm using novel variable forgetting factor with unity ZONE, Electronic Letter 27: 2150-2151.

Schmidt, D. \& Chen, T. (1986). Frequency domain synthesis of a robust flutter suppression control law, Journal of Guidance, Control, and Dynamics 9(3): 346-351.

Schmitt, N. P. Amd Rehm, W., Pistner, T., Zeller, P., Diehl, H. \& Navé, P. (2007). The AWAITOR airborne LIDAR turbulence sensor, Aerospace Science and Technology 11: 546-552.

Schmitt, N., Rehm, W., Pistner, T., Diehl, H. Navé, P., Jenaro-Rabadan, G., Mirand, P. \& Reymond, M. (2007). Flight test of the AWAITOR airborne LIDAR turbulence sensor, 14th Coherent Laser Radar Conference, Colorado.

Wildschek, A., Maier, R., Hoffmann, F., Jeanneau, M. \& Baier, H. (2006). Active wing load alleviation with an adaptive feedforward control algorithm, AIAA Guidance, Navigation, and Control Conference and Exhibit, Keyston, Colorado. AIAA-2006-6054.

Woods-Vedeler, J. A., Pototzky, A. \& Hoadley, S. T. (1995). Rolling maneuver load alleviation using active controls, Journal of Aircraft 32(1): 68-76.

Zeng, J. \& de Callafon, R. A. (2006). Model matching and filter design using orthonormal basis functions, 45th IEEE Conference on Decision and Control, San Diego, pp. 5347-5352.

Zeng, J. \& de Callafon, R. A. (2008). Adaptive Feedforward/Feedback control framework, Technical Report SBIR Phase I NNX08CB12P, ZONA Technology Inc. 


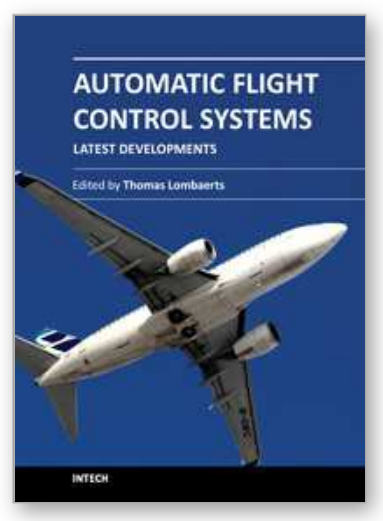

\author{
Automatic Flight Control Systems - Latest Developments \\ Edited by Dr. Thomas Lombaerts
}

ISBN 978-953-307-816-8

Hard cover, 204 pages

Publisher InTech

Published online 18, January, 2012

Published in print edition January, 2012

The history of flight control is inseparably linked to the history of aviation itself. Since the early days, the concept of automatic flight control systems has evolved from mechanical control systems to highly advanced automatic fly-by-wire flight control systems which can be found nowadays in military jets and civil airliners. Even today, many research efforts are made for the further development of these flight control systems in various aspects. Recent new developments in this field focus on a wealth of different aspects. This book focuses on a selection of key research areas, such as inertial navigation, control of unmanned aircraft and helicopters, trajectory control of an unmanned space re-entry vehicle, aeroservoelastic control, adaptive flight control, and fault tolerant flight control. This book consists of two major sections. The first section focuses on a literature review and some recent theoretical developments in flight control systems. The second section discusses some concepts of adaptive and fault-tolerant flight control systems. Each technique discussed in this book is illustrated by a relevant example.

\title{
How to reference
}

In order to correctly reference this scholarly work, feel free to copy and paste the following:

Jie Zeng, Raymond De Callafon and Martin J. Brenner (2012). Adaptive Feedforward Control for Gust Loads Alleviation, Automatic Flight Control Systems - Latest Developments, Dr. Thomas Lombaerts (Ed.), ISBN: 978953-307-816-8, InTech, Available from: http://www.intechopen.com/books/automatic-flight-control-systemslatest-developments/adaptive-feedforward-control-for-gust-loads-alleviation

\section{INTECH}

open science | open minds

\section{InTech Europe}

University Campus STeP Ri

Slavka Krautzeka 83/A

51000 Rijeka, Croatia

Phone: +385 (51) 770447

Fax: +385 (51) 686166

www.intechopen.com

\section{InTech China}

Unit 405, Office Block, Hotel Equatorial Shanghai

No.65, Yan An Road (West), Shanghai, 200040, China

中国上海市延安西路65号上海国际贵都大饭店办公楼 405 单元

Phone: +86-21-62489820

Fax: $+86-21-62489821$ 
(C) 2012 The Author(s). Licensee IntechOpen. This is an open access article distributed under the terms of the Creative Commons Attribution 3.0 License, which permits unrestricted use, distribution, and reproduction in any medium, provided the original work is properly cited. 